\title{
Parent Global Assessment of Disease
} Activity

National Cancer Institute

\section{Source}

National Cancer Institute. Parent Global Assessment of Disease Activity. NCI Thesaurus. Code C121347.

A parent-scored instrument that estimates the level of activity of a disease at a given time taking into account all disease manifestations. Typically scored on a numeric rating scale or visual analogue scale, or may be recorded using descriptors such as remission, or low, moderate, or high disease activity. 